\title{
Health Literacy, eHealth Literacy, Adherence to Infection Prevention and Control Procedures, Lifestyle Changes, and Suspected COVID-19 Symptoms Among Health Care Workers During Lockdown: Online Survey
}

Binh N Do ${ }^{1,2,3^{*}}$, MD, PhD; Tien V Tran ${ }^{1,2,3^{*}}$, MD, PhD; Dung T Phan ${ }^{4,5}$, RN, MPH, PhD; Hoang C Nguyen ${ }^{6,7}$, MD, $\mathrm{PhD}$; Thao T P Nguyen ${ }^{8,9}$, MD; Huu C Nguyen ${ }^{10,11}, \mathrm{MD}$, PhD; Tung H Ha ${ }^{12}, \mathrm{MD}, \mathrm{PhD}$; Hung K Dao ${ }^{13}, \mathrm{MD}, \mathrm{PhD}$; Manh V Trinh ${ }^{14}$, MD; Thinh V Do ${ }^{15}$, MD; Hung Q Nguyen ${ }^{16}$, MD, MSc; Tam T Vo ${ }^{17}$, MD; Nhan P T Nguyen ${ }^{18}$, MD, PhD; Cuong Q Tran ${ }^{19,20}$, MD, MPH, MBA; Khanh V Tran ${ }^{21}$, MD; Trang T Duong ${ }^{22}$, RN, MPH; Hai X Pham ${ }^{23}$, MD; Lam V Nguyen ${ }^{24,25}$, MD, PhD; Kien T Nguyen ${ }^{26}$, MA; Peter W S Chang ${ }^{27,28}$, MD, ScD; Tuyen Van Duong ${ }^{29}$, BNSc, MNSc, PhD

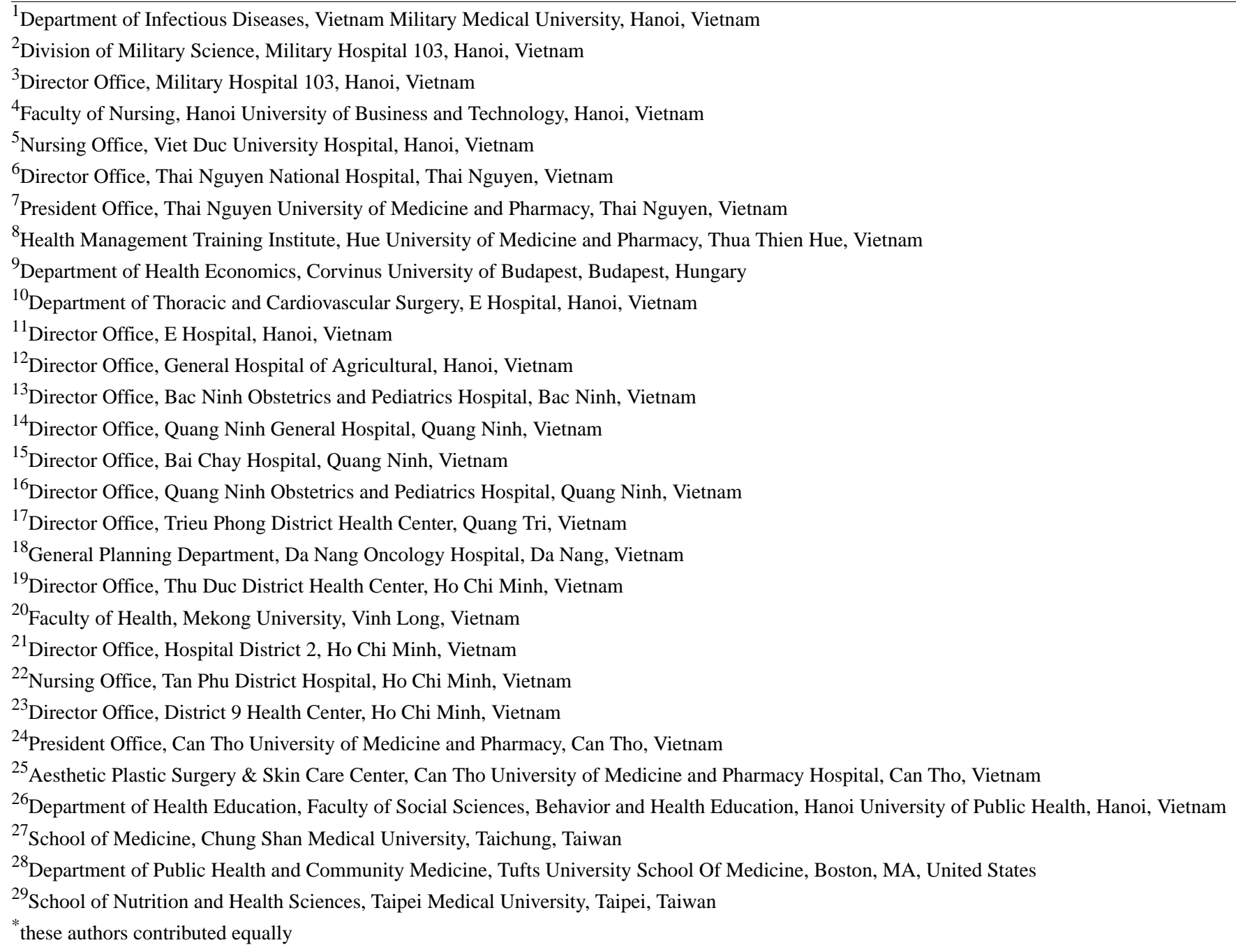

Corresponding Author:

Tuyen Van Duong, BNSc, MNSc, PhD

School of Nutrition and Health Sciences

Taipei Medical University

250 Wuxing St

Taipei, 11031 
Taiwan

Phone: 886227361661 ext 6545

Email: tvduong@tmu.edu.tw

\section{Abstract}

Background: The COVID-19 pandemic has imposed a heavy burden on health care systems and governments. Health literacy (HL) and eHealth literacy (as measured by the eHealth Literacy Scale [eHEALS]) are recognized as strategic public health elements but they have been underestimated during the pandemic. HL, eHEALS score, practices, lifestyles, and the health status of health care workers (HCWs) play crucial roles in containing the COVID-19 pandemic.

Objective: The aim of this study is to evaluate the psychometric properties of the eHEALS and examine associations of HL and eHEALS scores with adherence to infection prevention and control (IPC) procedures, lifestyle changes, and suspected COVID-19 symptoms among HCWs during lockdown.

Methods: We conducted an online survey of 5209 HCWs from 15 hospitals and health centers across Vietnam from April 6 to April 19, 2020. Participants answered questions related to sociodemographics, HL, eHEALS, adherence to IPC procedures, behavior changes in eating, smoking, drinking, and physical activity, and suspected COVID-19 symptoms. Principal component analysis, correlation analysis, and bivariate and multivariate linear and logistic regression models were used to validate the eHEALS and examine associations.

Results: The eHEALS had a satisfactory construct validity with 8 items highly loaded on one component, with factor loadings ranked from 0.78 to 0.92 explaining $76.34 \%$ of variance; satisfactory criterion validity as correlated with $\mathrm{HL}(\rho=0.42)$; satisfactory convergent validity with high item-scale correlations $(\rho=0.80-0.84)$; and high internal consistency (Cronbach $\alpha=.95)$. HL and eHEALS scores were significantly higher in men (unstandardized coefficient $[\mathrm{B}]=1.01,95 \% \mathrm{CI} 0.57-1.45, P<.001 ; \mathrm{B}=0.72,95 \%$ CI $0.43-1.00, P<.001)$, those with a better ability to pay for medication $(\mathrm{B}=1.65,95 \% \mathrm{CI} 1.25-2.05, P<.001 ; \mathrm{B}=0.60,95 \% \mathrm{CI}$ $0.34-0.86, P<.001)$, doctors $(\mathrm{B}=1.29,95 \%$ CI $0.73-1.84, P<.001 ; \mathrm{B} 0.56,95 \%$ CI $0.20-0.93, P=.003)$, and those with epidemic containment experience $(\mathrm{B}=1.96,95 \% \mathrm{CI} 1.56-2.37, P<.001 ; \mathrm{B}=0.64,95 \% \mathrm{CI} 0.38-0.91, P<.001)$, as compared to their counterparts, respectively. HCWs with higher HL or eHEALS scores had better adherence to IPC procedures $(\mathrm{B}=0.13,95 \% \mathrm{CI} 0.10-0.15$, $P<.001 ; \mathrm{B}=0.22,95 \% \mathrm{CI} 0.19-0.26, P<.001$ ), had a higher likelihood of healthy eating (odds ratio [OR] 1.04, 95\% CI 1.01-1.06, $P=.001$; OR $1.04,95 \%$ CI 1.02-1.07, $P=.002$ ), were more physically active (OR 1.03, 95\% CI 1.02-1.03, $P<.001 ;$ OR $1.04,95 \%$ CI 1.03-1.05, $P<.001)$, and had a lower likelihood of suspected COVID-19 symptoms (OR 0.97, 95\% CI 0.96-0.98, $P<.001$; OR $0.96,95 \%$ CI $0.95-0.98, P<.001$ ), respectively.

Conclusions: The eHEALS is a valid and reliable survey tool. Gender, ability to pay for medication, profession, and epidemic containment experience were independent predictors of HL and eHEALS scores. HCWs with higher HL or eHEALS scores had better adherence to IPC procedures, healthier lifestyles, and a lower likelihood of suspected COVID-19 symptoms. Efforts to improve HCWs' HL and eHEALS scores can help to contain the COVID-19 pandemic and minimize its consequences.

(J Med Internet Res 2020;22(11):e22894) doi: 10.2196/22894

\section{KEYWORDS}

COVID-19; health literacy; eHealth literacy; health care workers; personal protective equipment; handwashing; masks; disposing; lifestyle; Vietnam; eHealth; adherence; infection prevention; control

\section{Introduction}

COVID-19, the disease caused by SARS-CoV-2, has created unprecedented challenges worldwide [1-6], with significant socioeconomic burdens [7], morbidity, and mortality [8,9]. Multidisciplinary and multidimensional approaches are required to contain the pandemic [10-12]. Social and behavioral changes $[13,14]$ and improving health literacy (HL) [15] and eHealth literacy (as measured by the eHealth Literacy Scale [eHEALS]) [16] are highly recommended to control this global health crisis while effective treatments and vaccines are unavailable.

Lockdown measures were applied in many countries, including Vietnam [17]. Lockdown is a necessary public health approach to contain COVID-19. During the lockdown period, health care workers (HCWs) still needed to work and provide health services to patients, which made them more vulnerable to
COVID-19 infection. In addition, the pandemic itself and social distancing measures have had negative impacts on psychological health $[18,19]$ and health-related behavior (eg, unhealthy eating habits and behaviors, less physical activity, and more smoking [20-23] and drinking to cope amid the COVID-19 pandemic [24]).

Online consultations from hospitals and health care centers were found to be a safe and effective way to reduce the negative effects of the pandemic [25]. In addition, social media can provide insights for effective communication among public health authorities [26], researchers [27], and the public [28-30]. However, social media spreads panic and anxiety among the public [31]. Disinformation and misinformation have been raised as highly concerning issues for the public [32-34]. HL plays an important role in evaluating online health information [35]. HL is critical for people who interact with the digital world, with 
its diverse information and sources [36], especially during the COVID-19 pandemic. HCWs play an important role in supporting the public to combat misinformation and disinformation $[28,37]$. The HCWs' consultations cannot be altered by social media networks $[37,38]$. Continuous training and education have been recognized as effective approaches to improving HCWs' HL, further improving health care delivery $[39,40]$, communication $[39,41,42]$, shared decision making [43], and patient health outcomes [40]. Furthermore, raising awareness of behavioral pitfalls could support appropriate behavioral changes and containment of the crisis [44]. HL and eHealth literacy are more important than ever due to the COVID-19 pandemic [16,45]. However, these issues have been underestimated during the pandemic [15].

We evaluated the psychometric properties of the eHEALS and examined the predictors of HL and eHEALS scores. We also examined the associations between HL and eHEALS scores with adherence to infection prevention and control (IPC) measures, lifestyle changes, and suspected COVID-19 symptoms among HCWs during the lockdown period in Vietnam.

\section{Methods}

\section{Study Design and Settings}

A cross-sectional study was conducted with HCWs April 6-19, 2020, using online-based questionnaires (Text 1 in Multimedia Appendix 1). The HCWs were recruited from 12 hospitals and 3 health centers across Vietnam, including 8 hospitals in the nation's north, 1 hospital and 1 health center in the central region, and 3 hospitals and 2 health centers in the country's south.

\section{Study Participants and Data Collection}

No HCWs (doctors and nurses) in our study had provided any direct care or had contact with patients with COVID-19. A total sample of $5209 \mathrm{HCWs}$ (out of 11,517 possible participants) completed an online survey. The studied and possible participants from public hospitals and health centers are presented in Table 1. 
Table 1. Participants from the studied hospitals and health centers by geographic location.

\begin{tabular}{|c|c|c|}
\hline Geographic location and hospital/health center & Possible participants & Studied participants \\
\hline \multicolumn{3}{|l|}{ North } \\
\hline \multicolumn{3}{|l|}{ Ha Noi city } \\
\hline Military Hospital 103 & 1660 & 177 \\
\hline E hospital & 1125 & 335 \\
\hline General Hospital of Agricultural & 555 & 424 \\
\hline \multicolumn{3}{|l|}{ Thai Nguyen province } \\
\hline Thai Nguyen National Hospital & 1186 & 988 \\
\hline \multicolumn{3}{|l|}{ Bac Ninh city } \\
\hline Bac Ninh Obstetrics and Pediatrics Hospital & 391 & 364 \\
\hline \multicolumn{3}{|l|}{ Quang Ninh province } \\
\hline Quang Ninh General Hospital & 922 & 675 \\
\hline Bai Chay Hospital & 819 & 476 \\
\hline Quang Ninh Obstetrics and Pediatrics Hospital & 478 & 290 \\
\hline \multicolumn{3}{|l|}{ Center } \\
\hline \multicolumn{3}{|l|}{ Quang Tri province } \\
\hline Trieu Phong District Health Center & 271 & 203 \\
\hline \multicolumn{3}{|l|}{ Da Nang city } \\
\hline Da Nang Oncology Hospital & 555 & 134 \\
\hline \multicolumn{3}{|l|}{ South } \\
\hline \multicolumn{3}{|l|}{ Ho Chi Minh city } \\
\hline Tan Phu District Hospital & 530 & 241 \\
\hline Hospital District 2 & 812 & 318 \\
\hline District 9 Health Center & 170 & 102 \\
\hline Thu Duc District Health Center & 302 & 291 \\
\hline \multicolumn{3}{|l|}{ Can Tho city } \\
\hline Can Tho University of Medicine and Pharmacy Hospital & 424 & 191 \\
\hline Total & 11,517 & 5209 \\
\hline
\end{tabular}

Vietnam applied a nationwide lockdown measure April 1-15, 2020 [17], which was extended to April 22, 2020 [46,47]. In this study, HCWs took the online survey during the lockdown period. We used Google Forms to design and conduct the survey. The online survey links were sent to HCWs by researchers via email, Messenger, or Zalo. QR codes were also displayed in different departments of hospitals and health centers. It took about 15 minutes to complete the questionnaire. All survey questions were mandatory; therefore, there is no missing data in our study. All responses were exported to Google Sheets and saved on Google Drive. Finally, the data was coded, cleaned, and analyzed confidentially by researchers.

\section{Measures}

\section{Sociodemographics}

HCWs reported their age (21-40 years versus 41-60 years), gender (woman versus man), marital status (never married versus ever married), ability to pay for medication (very or fairly difficult versus very or fairly easy), social status (low versus middle to high), profession (doctor, nurse, or other, the last of which included medical technicians, midwives, pharmacists, pharmacy technicians, administrative staff, catering staff, and cleaners), type of health care facility (second-line versus frontline, the latter of which includes the outpatient department, emergency department, quarantine and isolation areas, medical imaging and laboratory diagnosis department, and patient administration areas), and previous epidemic (eg, SARS, tuberculosis, influenza A) containment experience (no versus yes). Additionally, comorbidity was assessed using the Charlson comorbidity index items $[48,49]$.

\section{Health Literacy}

A 12-item short-form health literacy questionnaire (HLS-SF12) was used. The questionnaire was validated and used in Asian countries [50], including Vietnam [51-54]. HCWs rated their perceived difficulty of items based on a 4-point Likert scale from $1=$ Very difficult to $4=$ Very easy. The health literacy index score was standardized to an unified metric from 0 to 50, with higher scores representing better health literacy $[50,55]$. 


\section{eHealth Literacy}

The widely used eHealth literacy scale (eHEALS) with 8 items was used to assess HCWs' eHealth literacy skills [56]. The questionnaire was translated into Vietnamese by the researchers. The content was then validated by an expert panel (28 medical doctors, 7 nurses, and 9 nutrition and public health professionals). The expert panel suggested retaining the original rating and scoring system. HCWs rated their experiences using the internet for health information based on a 5-point Likert scale from $1=$ Strongly disagree to $5=$ Strongly agree. Scores range from 8 to 40 , with high eHEALS scores representing better eHealth literacy.

\section{Adherence to IPC Procedures}

Participants reported the practices and activities performed related to COVID-19 IPC during health care interactions. The questionnaire was adapted from the interim guidance of the World Health Organization [57]. The IPC measures included the following: (1) wearing single-use gloves, (2) wearing a medical mask, (3) wearing a face shield or goggles/protective glasses, (4) wearing a disposable gown, (5) removing and replacing personal protective equipment (PPE) according to protocol, (6) performing hand hygiene before and after touching patients, (7) performing hand hygiene before and after performing any clean or aseptic procedure, (8) performing hand hygiene after exposure to body fluid, (9) performing hand hygiene after touching patients' surroundings, and (10) decontaminating high-touch surfaces. HCWs quantified the frequency of performance of the above IPC procedures as recommended, where $0=$ Never, $1=$ Rarely $(<20 \%$ of the time), $2=$ Occasionally ( $20 \%$ to $<50 \%$ of the time), $3=$ Most of the time ( $50 \%$ to $<95 \%$ of the time), and $4=$ Always ( $\geq 95 \%$ of the time). The total performance score of the 10 activities ranged from 0 to 40 , with higher scores indicating better adherence to IPC procedures.

\section{Lifestyle Changes}

HCWs reported their current smoking (never/stopped/less versus unchanged or more), drinking (never/stopped/less versus unchanged or more), physical activity (never/stopped/less versus unchanged or more), and eating (less healthy versus unchanged or healthier) behaviors as compared with that before the pandemic [54].

\section{Suspected COVID-19 Symptoms}

HCWs were screened for suspected COVID-19 symptoms [58], including the common symptoms of fever, cough, and dyspnea, along with the less common symptoms of myalgia, fatigue, sputum production, confusion, headache, sore throat, rhinorrhea, chest pain, hemoptysis, diarrhea, and nausea/vomiting. HCWs with any of those symptoms were classified as having symptoms indicative of COVID-19.

\section{Ethical Consideration}

The study was reviewed and approved by the Institutional Ethical Review Committee of Hanoi University of Public Health, Vietnam (Institutional Review Board number 133/2020/YTCC-HD3). The HCWs voluntarily took the survey.

\section{Data Analysis}

\section{Psychometric Properties of the eHEALS}

\section{Construct Validity}

The construct validity of the eHEALS was examined using principal component analysis (PCA). An Kaiser-Meyer Olkin (KMO) value $\geq 0.6$ was set to measure sampling adequacy and a Bartlett Test of Sphericity value $<0.05$ was set to determine the suitability of the data for PCA [59]. The oblique rotation (Promax) method was used.

\section{Convergent Validity}

The Spearman correlation was used to check the correlations between the eHEALS and its eight items.

\section{Criterion Validity}

The Pearson correlation between eHEALS and HLS-SF12 was estimated to provide evidence of criterion validity [60].

\section{Floor and Ceiling Effects}

The percentages of the lowest and highest score among HCWs were calculated. Minimal percentages $(<15 \%)$ were recommended to eliminate floor and ceiling effects [61].

\section{Reliability Analysis}

The internal consistency of the eHEALS was estimated using the Cronbach $\alpha$ test. A Cronbach $\alpha$ value $\geq .70$ was designated as satisfactory reliability [62].

\section{Health Literacy, eHealth Literacy, and Associated Factors}

The distributions of HL and eHEALS scores in different categories of studied variables were explored using a one-way analysis of variance (ANOVA) test. In addition, bivariate and multivariate linear regression models were used to examine predictors of HL and eHEALS scores and to investigate the associations of HL and eHEALS scores with adherence to IPC measures. Next, bivariate and multivariate logistic regression models were used to examine the associations of HL and eHEALS scores with lifestyle changes and suspected COVID-19 symptoms. The factors that demonstrated associations with outcome variables at $P$ values $<.20$ in the bivariate models were included in multivariate models [63]. To exclude colliders, which may cause multicollinearity, we checked the correlations between them using the Spearman correlation test. The representative factors were selected in the multivariate analysis. The regression coefficient (B), odds ratio (OR), and 95\% confidence interval $(95 \% \mathrm{CI})$ were reported appropriately.

Data were analyzed using IBM SPSS (Version 20.0; IBM Corp). The significance level was set at a $P$ value $<.05$.

\section{Results}

\section{Characteristics of $\mathrm{HCWs}$}

Table 2 indicates that, of 5209 HCWs, $905(17.4 \%)$ were aged 41-60 years, $1714(32.9 \%)$ were men, 3915 (75.2\%) were ever married, $2458(47.2 \%)$ could very or fairly easily pay for medication, $4506(86.5 \%)$ had a middle or high social status, $2540(48.8 \%)$ were nurses, 1453 (27.9\%) were doctors, 2299 
(44.1\%) were frontline HCWs, 1999 (38.4\%) had epidemic containment experience, $270(5.2 \%)$ had comorbidities, 769 (14.8\%) had had suspected COVID-19 symptoms, 5042 (96.8\%) ate at an "unchanged or healthier" level, 228 (4.4\%) smoked at an "unchanged or more" level, $234(4.5 \%)$ drank at an "unchanged or more" level, and 3553 (68.2\%) had an "unchanged or more" physical activity level during the pandemic. The mean (SD) scores of adherence to IPC measures, HL, and eHEALS were 30.6 (6.2), 36.2 (7.3), and 33.1 (4.8), respectively. The HL scores varied by age, gender, ability to pay for medication, social status, type of health care personnel, epidemic containment experience, BMI, suspected COVID-19 symptoms, dietary intake, smoking, and physical activity $(P<.05)$. The eHEALS scores varied by gender, ability to pay for medication, type of health care personnel, epidemic containment experience, BMI, suspected COVID-19 symptoms, dietary intake, smoking, and physical activity (Table 2). 
Table 2. Characteristics, health literacy, and eHealth literacy among health care workers.

\begin{tabular}{|c|c|c|c|c|c|}
\hline \multirow[t]{2}{*}{ Variables } & \multirow{2}{*}{$\begin{array}{l}\text { Total }(\mathrm{N}=5209) \\
\text { Participants, n (\%) }\end{array}$} & \multicolumn{2}{|l|}{ Health literacy } & \multicolumn{2}{|l|}{$\begin{array}{l}\text { eHealth Literacy } \\
\text { Scale }\end{array}$} \\
\hline & & Mean (SD) & $P$ value $^{\mathrm{a}}$ & Mean (SD) & $P$ value $^{\mathrm{a}}$ \\
\hline Age (years) & & & .02 & $\mathrm{~N} / \mathrm{A}^{\mathrm{b}}$ & .88 \\
\hline $21-40$ & $4304(82.6)$ & $36.1(7.2)$ & N/A & $33.1(4.7)$ & N/A \\
\hline $41-60$ & 905 (17.4) & $36.7(7.5)$ & N/A & $33.1(5.0)$ & N/A \\
\hline Gender & & & $<.001$ & N/A & $<.001$ \\
\hline Women & $3495(67.1)$ & $35.7(7.0)$ & N/A & $32.8(4.5)$ & N/A \\
\hline Men & $1714(32.9)$ & $37.1(7.8)$ & N/A & $33.8(5.3)$ & N/A \\
\hline Marital status & & & .04 & N/A & .29 \\
\hline Never married & $1294(24.8)$ & $35.8(7.0)$ & N/A & $33.0(4.8)$ & N/A \\
\hline Ever married & 3915 (75.2) & $36.3(7.4)$ & N/A & $33.2(4.7)$ & N/A \\
\hline Ability to pay for medication & & & $<.001$ & N/A & $<.001$ \\
\hline Very or fairly difficult & $2751(52.8)$ & $35.3(7.4)$ & N/A & $32.8(4.9)$ & N/A \\
\hline Very or fairly easy & $2458(47.2)$ & $37.2(7.0)$ & N/A & $33.5(4.6)$ & N/A \\
\hline Social status & & & $<.001$ & N/A & .27 \\
\hline Low & $703(13.5)$ & $35.0(7.7)$ & N/A & $32.9(4.7)$ & N/A \\
\hline Middle or high & $4506(86.5)$ & $36.3(7.2)$ & N/A & $33.2(4.8)$ & N/A \\
\hline Type of health care personnel & & & $<.001$ & N/A & $<.001$ \\
\hline Other & $1216(23.3)$ & $35.7(7.6)$ & N/A & $33.1(5.1)$ & N/A \\
\hline Nurse & $2540(48.8)$ & $35.7(7.2)$ & N/A & $32.7(4.6)$ & N/A \\
\hline Doctor & $1453(27.9)$ & $37.3(7.1)$ & N/A & $33.9(4.8)$ & N/A \\
\hline Type of health care facility & & & .77 & N/A & .46 \\
\hline Non-frontline & $2910(55.9)$ & $36.2(7.4)$ & N/A & $33.2(4.7)$ & \\
\hline Frontline & $2299(44.1)$ & $36.1(7.1)$ & N/A & $33.1(4.8)$ & N/A \\
\hline Epidemic containment experience & & & $<.001$ & N/A & $<.001$ \\
\hline No & $3210(61.6)$ & $35.4(7.2)$ & N/A & $32.9(4.6)$ & N/A \\
\hline Yes & $1999(38.4)$ & $37.4(7.3)$ & N/A & $33.5(5.0)$ & N/A \\
\hline Comorbidity & & & .38 & N/A & .37 \\
\hline None & $4939(94.8)$ & $36.1(7.3)$ & N/A & $33.1(4.8)$ & N/A \\
\hline One or more & $270(5.2)$ & $36.5(7.6)$ & N/A & $32.9(5.0)$ & N/A \\
\hline Suspected COVID-19 symptoms ${ }^{d}$ & & & $<.001$ & N/A & $<.001$ \\
\hline No & $4440(85.2)$ & $36.4(7.3)$ & N/A & $33.3(4.7)$ & N/A \\
\hline Yes & $769(14.8)$ & $34.7(7.2)$ & N/A & $32.3(5.1)$ & N/A \\
\hline Dietary intake $^{\mathrm{e}}$ & & & $<.001$ & N/A & .002 \\
\hline Eat less healthy & $167(3.2)$ & $34.2(6.9)$ & N/A & $32.0(5.9)$ & N/A \\
\hline Unchanged or healthier & $5042(96.8)$ & $36.2(7.3)$ & N/A & $33.2(4.7)$ & N/A \\
\hline Smoking tobacco $f$ & & & .046 & N/A & .03 \\
\hline Never, stopped, or less & $4981(95.6)$ & $36.1(7.2)$ & N/A & $33.1(4.7)$ & N/A \\
\hline Unchanged or more & $228(4.4)$ & $37.1(8.6)$ & N/A & $33.8(5.5)$ & N/A \\
\hline Drinking alcohol $\mathbf{f}$ & & & .18 & N/A & .53 \\
\hline Never, stopped, or less & $4975(95.5)$ & $36.1(7.2)$ & N/A & $33.1(4.7)$ & N/A \\
\hline
\end{tabular}




\begin{tabular}{|c|c|c|c|c|c|}
\hline \multirow[t]{2}{*}{ Variables } & \multirow{2}{*}{$\begin{array}{l}\text { Total (N=5209) } \\
\text { Participants, n (\%) }\end{array}$} & \multicolumn{2}{|c|}{ Health literacy } & \multicolumn{2}{|l|}{$\begin{array}{l}\text { eHealth Literacy } \\
\text { Scale }\end{array}$} \\
\hline & & Mean (SD) & $P$ value $^{\mathrm{a}}$ & Mean (SD) & $P$ value $^{\mathrm{a}}$ \\
\hline Unchanged or more & $234(4.5)$ & $36.8(8.3)$ & N/A & $33.3(5.7)$ & N/A \\
\hline Physical activity ${ }^{\mathbf{f}}$ & & & $<.001$ & N/A & $<.001$ \\
\hline Never, stopped, or less & $1656(31.8)$ & $35.2(7.6)$ & N/A & $32.5(5.2)$ & N/A \\
\hline Unchanged or more & $3553(68.2)$ & $36.6(7.1)$ & N/A & $33.4(4.6)$ & N/A \\
\hline $\begin{array}{l}\text { Adherence to infection prevention and control } \\
\text { measures, mean (SD) }\end{array}$ & $30.6(6.2)$ & N/A & N/A & N/A & N/A \\
\hline Health literacy index, mean (SD) & $36.2(7.3)$ & N/A & N/A & N/A & N/A \\
\hline eHealth Literacy Scale score, mean (SD) & $33.1(4.8)$ & N/A & N/A & N/A & N/A \\
\hline
\end{tabular}

${ }^{\mathrm{a}} P$ value: result of one-way analysis of variance (ANOVA) test.

${ }^{\mathrm{b}} \mathrm{N} / \mathrm{A}$ : not applicable.

${ }^{c}$ Frontline areas include the outpatient department, emergency department, isolation areas, imaging and laboratory diagnosis department, and patient administration areas.

${ }^{\mathrm{d}}$ Suspected COVID-19 symptoms include common symptoms (fever, cough, dyspnea) and less common symptoms (myalgia, fatigue, sputum production, confusion, headache, sore throat, rhinorrhea, chest pain, hemoptysis, diarrhea, and nausea/vomiting).

${ }^{\mathrm{f}}$ Health care workers were asked whether their lifestyle behaviors got worse, better, or were unchanged during the COVID-19 pandemic as compared to before the pandemic.

\section{Psychometric Properties of eHealth Literacy}

As shown in Table 3, the KMO value of the overall scale was 0.93 and the values of its 8 items ranged from 0.92 to 0.95 . The Bartlett Test of Sphericity value was $<0.001$, which is a satisfactory level. In addition, the average communality value of 0.76 was satisfactory, demonstrating the accuracy of the approach [59]. Overall, the 8 items of the eHEALS were strongly loaded on one component and explained $76.34 \%$ of the scale variance. The factor loading values of 8 items were ranked from 0.78 to 0.92 , as shown in Table 3 . The correlations between each item and the scale range from 0.80 to 0.84 , indicating satisfactory convergent validity [50,51]. In addition, the correlation between eHEALS and HL scores was moderate $(\rho=0.42)$, providing evidence of criterion validity [60]. Furthermore, the Cronbach $\alpha$ value of .95 indicated a high level of internal consistency. There was no significant floor effect, with $0.70 \%$ of participants having the lowest potential response. A marginal ceiling effect was found, with $16.1 \%$ of participants having the highest potential response (slightly higher than the recommended percentage of $<15 \%$; Table 3 ). 
Table 3. Construct, convergent, and criterion validity, internal consistency, and floor and ceiling effects of the 8-item eHealth Literacy Scale (N=5209).

\begin{tabular}{ll}
\hline Construct validity, factor loadings & Values \\
\hline eHealth Literacy scale items & 0.85 \\
$\quad$ I know what health resources are available on the internet & 0.90 \\
I know where to find helpful health resources on the internet & 0.92 \\
I know how to find helpful health resources on the internet & 0.90 \\
$\quad$ I know how to use the internet to answer my questions about health & 0.92 \\
$\quad$ I know how to use the health information I find on the internet to help me & 0.89 \\
$\quad$ I have the skills I need to evaluate the health resources I find on the internet & 0.82 \\
$\quad$ I have the skills needed to tell high-quality health resources from low-quality health resources on the internet & 0.78 \\
$\quad$ I feel confident in using information from the internet to make health decisions & 76.34 \\
Percentage of variance, $\%$ & 0.83 (0.80-0.84) \\
Item-scale convergent validity, mean of $\rho^{\mathrm{a}}$ (range) & 0.42 \\
Criterion validity, correlation with health literacy, $\rho^{\mathrm{b}}$ & .95 \\
Internal consistency, Cronbach $\alpha$ & 0.70 \\
Floor effect, $\%$ & 16.10 \\
Ceiling effect, $\%$ & \\
\hline
\end{tabular}

${ }^{\mathrm{a}} \rho$ : Spearman correlation coefficient.

$\mathrm{b} \rho$ : Pearson correlation coefficient.

\section{Determinants of HL and eHealth Literacy}

The week correlations among independent variables $(\rho<0.30)$ suggest that there is no collider which might affect the results (Table 1 in Multimedia Appendix 1). Results of the multivariate analysis shown in Table 4 indicated that HCWs with higher HL scores were men (unstandardized regression coefficient $[\mathrm{B}]=1.01,95 \% \mathrm{CI} 0.57-1.45, P<.001)$, those with a very or fairly easy ability to pay for medication $(B=1.65,95 \%$ CI $1.25-2.05$, $P<.001)$, those with a middle or high social status $(\mathrm{B}=0.586$, 95\% CI $0.003-1.169, P=.049)$, doctors $(B=1.29,95 \%$ CI
$0.73-1.84, P<.001)$, and those with epidemic containment experience ( $\mathrm{B}=1.96,95 \% \mathrm{CI} 1.56-2.37, P<.001)$, as compared to their counterparts, respectively.

HCWs with higher eHEALS scores were men $(B=0.72,95 \%$ CI $0.43-1.00, P<.001$ ), those with a very or fairly easy ability to pay for medication $(\mathrm{B}=0.60,95 \%$ CI $0.34-0.86, P<.001)$, doctors $(\mathrm{B}=0.56,95 \% \mathrm{CI} 0.20-0.93, P=.003)$, and those with epidemic containment experience $(\mathrm{B}=0.64,95 \%$ CI $0.38-0.91$, $P<.001$ ), as compared to their counterparts, respectively (Table 4). 
Table 4. Determinants of health literacy and eHealth literacy among health care workers $(\mathrm{N}=5209)$.

\begin{tabular}{|c|c|c|c|c|c|c|c|c|}
\hline \multirow[t]{3}{*}{ Variables } & \multicolumn{4}{|l|}{ Health literacy } & \multicolumn{4}{|l|}{ eHealth literacy } \\
\hline & \multicolumn{2}{|l|}{ Bivariate } & \multicolumn{2}{|l|}{ Multivariate } & \multicolumn{2}{|l|}{ Bivariate } & \multicolumn{2}{|l|}{ Multivariate } \\
\hline & $\mathrm{B}^{\mathrm{a}}(95 \% \mathrm{CI})$ & $P$ value & $\mathrm{B}(95 \% \mathrm{CI})$ & $P$ value & $\mathrm{B}(95 \% \mathrm{CI})$ & $P$ value & $\mathrm{B}(95 \% \mathrm{CI})$ & $P$ value \\
\hline \multicolumn{9}{|l|}{ Age (years) } \\
\hline $21-40$ & Reference & $\mathrm{N} / \mathrm{A}^{\mathrm{b}}$ & Reference & N/A & Reference & N/A & Reference & N/A \\
\hline $41-60$ & $\begin{array}{l}0.62(0.10 \text { to } \\
1.14)\end{array}$ & .02 & $\begin{array}{l}-0.05(-0.58 \text { to } \\
0.49)\end{array}$ & .86 & $\begin{array}{l}-0.03(-0.37 \text { to } \\
0.31)\end{array}$ & .88 & $\begin{array}{l}-0.30(-0.65 \text { to } \\
0.04)\end{array}$ & .08 \\
\hline \multicolumn{9}{|l|}{ Gender } \\
\hline Female & Reference & N/A & Reference & N/A & Reference & N/A & Reference & N/A \\
\hline Male & $\begin{array}{l}1.36(0.94 \text { to } \\
1.78)\end{array}$ & $<.001$ & $\begin{array}{l}1.01(0.57 \text { to } \\
1.45)\end{array}$ & $<.001$ & $\begin{array}{l}0.99(0.72 \text { to } \\
1.27)\end{array}$ & $<.001$ & $\begin{array}{l}0.72(0.43 \text { to } \\
1.00)\end{array}$ & $<.001$ \\
\hline \multicolumn{9}{|l|}{ Marital status } \\
\hline Never married & Reference & N/A & Reference & N/A & Reference & N/A & N/A & N/A \\
\hline Ever married & $\begin{array}{l}0.47(0.01 \text { to } \\
0.93)\end{array}$ & .04 & $\begin{array}{l}0.34(-0.12 \text { to } \\
0.81)\end{array}$ & .15 & $\begin{array}{l}0.16(-0.14 \text { to } \\
0.46)\end{array}$ & .29 & N/A & N/A \\
\hline \multicolumn{9}{|c|}{ Ability to pay for medication } \\
\hline $\begin{array}{l}\text { Very or fairly dif- } \\
\text { ficult }\end{array}$ & Reference & N/A & Reference & $\mathrm{N} / \mathrm{A}$ & Reference & N/A & Reference & N/A \\
\hline $\begin{array}{l}\text { Very or fairly } \\
\text { easy }\end{array}$ & $\begin{array}{l}1.9(1.51 \text { to } \\
2.29)\end{array}$ & $<.001$ & $\begin{array}{l}1.65(1.25 \text { to } \\
2.05)\end{array}$ & $<.001$ & $\begin{array}{l}0.72(0.46 \text { to } \\
0.98)\end{array}$ & $<.001$ & $\begin{array}{l}0.60(0.34 \text { to } \\
0.86)\end{array}$ & $<.001$ \\
\hline \multicolumn{9}{|l|}{ Social status } \\
\hline Low & Reference & N/A & Reference & $\mathrm{N} / \mathrm{A}$ & Reference & N/A & N/A & N/A \\
\hline Middle or high & $\begin{array}{l}1.29(0.72 \text { to } \\
1.87)\end{array}$ & $<.001$ & $\begin{array}{l}0.586(0.003 \text { to } \\
1.169)\end{array}$ & .049 & $\begin{array}{l}0.22(-0.16 \text { to } \\
0.59)\end{array}$ & .27 & N/A & N/A \\
\hline \multicolumn{9}{|c|}{ Type of health care personnel } \\
\hline Other & Reference & N/A & Reference & N/A & Reference & $\mathrm{N} / \mathrm{A}$ & Reference & N/A \\
\hline Nurse & $\begin{array}{l}0.09(-0.41 \text { to } \\
0.58)\end{array}$ & .73 & $\begin{array}{l}0.18(-0.32 \text { to } \\
0.68)\end{array}$ & .48 & $\begin{array}{l}-0.40(-0.72 \text { to } \\
-0.07)\end{array}$ & .02 & $\begin{array}{l}-0.32(-0.65 \text { to } \\
0.01)\end{array}$ & .06 \\
\hline Doctor & $\begin{array}{l}1.68(1.13 \text { to } \\
2.24)\end{array}$ & $<.001$ & $\begin{array}{l}1.29(0.73 \text { to } \\
1.84)\end{array}$ & $<.001$ & $\begin{array}{l}0.76(0.39 \text { to } \\
1.12)\end{array}$ & $<.001$ & $\begin{array}{l}0.56(0.20 \text { to } \\
0.93)\end{array}$ & .003 \\
\hline \multicolumn{9}{|c|}{ Type of health care facility ${ }^{c}$} \\
\hline Non-frontline & Reference & N/A & N/A & N/A & Reference & $\mathrm{N} / \mathrm{A}$ & N/A & N/A \\
\hline Frontline & $\begin{array}{l}-0.06(-0.46 \text { to } \\
0.34)\end{array}$ & .77 & $\mathrm{~N} / \mathrm{A}$ & N/A & $\begin{array}{l}-0.10(-0.36 \text { to } \\
0.16)\end{array}$ & .46 & N/A & N/A \\
\hline \multicolumn{9}{|c|}{ Epidemic containment experience } \\
\hline No & Reference & N/A & Reference & N/A & Reference & N/A & Reference & N/A \\
\hline Yes & $\begin{array}{l}1.95(1.54 \text { to } \\
2.35)\end{array}$ & $<.001$ & $\begin{array}{l}1.96(1.56 \text { to } \\
2.37)\end{array}$ & $<.001$ & $\begin{array}{l}0.55(0.29 \text { to } \\
0.82)\end{array}$ & $<.001$ & $\begin{array}{l}0.64(0.38 \text { to } \\
0.91)\end{array}$ & $<.001$ \\
\hline \multicolumn{9}{|l|}{ Comorbidity } \\
\hline None & Reference & N/A & Reference & N/A & Reference & N/A & N/A & N/A \\
\hline One or more & $\begin{array}{l}0.40(-0.49 \text { to } \\
1.29)\end{array}$ & .378 & $\mathrm{~N} / \mathrm{A}$ & N/A & $\begin{array}{l}-0.27(-0.85 \text { to } \\
0.32)\end{array}$ & .369 & N/A & N/A \\
\hline
\end{tabular}

${ }^{\mathrm{a}} \mathrm{B}$ : unstandardized regression coefficient.

${ }^{\mathrm{b}}$ N/A: not applicable.

${ }^{\mathrm{c}}$ Frontline areas are the outpatient department, emergency department, isolation areas, imaging and laboratory diagnosis department, and patient administration areas. 


\section{Association Between HL and eHealth Literacy and Adherence to IPC Procedures}

According to the results of a multivariate linear regression analysis (Table 5), higher HL $(\mathrm{B}=0.13$, 95\% CI $0.10-0.15$, $P<.001)$ and eHEALS scores $(\mathrm{B}=0.22$, 95\% CI $0.19-0.26$,
$P<.001)$ were found to be associated with better adherence to IPC measures after adjusting for age, gender, ability to pay for medication, social status, profession, type of health care facility, epidemic containment experience, and comorbidities. These adjusted factors showed the associations with adherence to IPC measures at $P<.20$ (Table 2 in Multimedia Appendix 1).

Table 5. Associations of health literacy and eHealth literacy with adherence to infection prevention and control measures, lifestyle changes, and suspected COVID-19 symptoms among health care workers ( $\mathrm{N}=5209)$.

\begin{tabular}{|c|c|c|c|c|c|c|c|c|c|c|c|c|}
\hline \multirow[t]{2}{*}{ Variables } & \multicolumn{2}{|l|}{$\begin{array}{l}\text { Adher- } \\
\text { ence to } \\
\text { IPC mea- } \\
\text { sures }^{\mathrm{a}}\end{array}$} & \multicolumn{2}{|l|}{$\begin{array}{l}\text { Dietary } \\
\text { intake }^{b}\end{array}$} & \multicolumn{2}{|l|}{$\begin{array}{l}\text { Smoking } \\
\text { tobacco }^{c}\end{array}$} & \multicolumn{2}{|l|}{$\begin{array}{l}\text { Drinking } \\
\text { alcohol }^{\mathrm{d}}\end{array}$} & \multicolumn{2}{|l|}{$\begin{array}{l}\text { Physical } \\
\text { activity }^{\mathrm{e}}\end{array}$} & \multicolumn{2}{|l|}{$\begin{array}{l}\text { Suspect- } \\
\text { ed } \\
\text { COVID- } \\
19 \text { symp- } \\
\text { toms }\end{array}$} \\
\hline & $\begin{array}{l}\mathrm{B}^{\mathrm{g}}(95 \% \\
\mathrm{CI})\end{array}$ & $\begin{array}{l}P \\
\text { val- } \\
\text { ue }\end{array}$ & $\begin{array}{l}\mathrm{OR}^{\mathrm{h}} \\
(95 \% \mathrm{CI})\end{array}$ & $\begin{array}{l}P \\
\text { val- } \\
\text { ue }\end{array}$ & $\begin{array}{l}\text { OR }(95 \% \\
\text { CI })\end{array}$ & $P$ value & $\begin{array}{l}\text { OR }(95 \% \\
\text { CI) }\end{array}$ & $\begin{array}{l}P \\
\text { val- } \\
\text { ue }\end{array}$ & $\begin{array}{l}\text { OR }(95 \% \\
\text { CI) }\end{array}$ & $P$ value & $\begin{array}{l}\text { OR }(95 \% \\
\text { CI })\end{array}$ & $\begin{array}{l}P \\
\text { val- } \\
\text { ue }\end{array}$ \\
\hline \multicolumn{13}{|c|}{ Health literacy, 1-score increment } \\
\hline Bivariate model & $\begin{array}{l}0.13 \\
(0.11- \\
0.15)\end{array}$ & $<.001$ & $\begin{array}{l}1.04 \\
(1.02- \\
1.06)\end{array}$ & $<.001$ & $\begin{array}{l}1.02(1.00- \\
1.04)\end{array}$ & .046 & $\begin{array}{l}1.01(0.99- \\
1.03)\end{array}$ & .18 & $\begin{array}{l}1.03(1.02- \\
1.04)\end{array}$ & $<.001$ & $\begin{array}{l}0.97 \\
(0.96- \\
0.98)\end{array}$ & $<.001$ \\
\hline $\begin{array}{l}\text { Multivariate } \\
\text { model }\end{array}$ & $\begin{array}{l}0.13 \\
(0.10- \\
0.15)\end{array}$ & $<.001$ & $\begin{array}{l}1.04 \\
(1.01- \\
1.06)\end{array}$ & .001 & $\begin{array}{l}1.01(0.99- \\
1.03)\end{array}$ & .34 & $\begin{array}{l}1.01(0.99- \\
1.02)\end{array}$ & .47 & $\begin{array}{l}1.03(1.02- \\
1.03)\end{array}$ & $<.001$ & $\begin{array}{l}0.97 \\
(0.96- \\
0.98)\end{array}$ & $<.001$ \\
\hline \multicolumn{13}{|c|}{ eHealth Literacy Scale, 1-score increment } \\
\hline Bivariate model & $\begin{array}{l}0.22 \\
(0.18- \\
0.25)\end{array}$ & $<.001$ & $\begin{array}{l}1.04 \\
(1.02- \\
1.07)\end{array}$ & .002 & $\begin{array}{l}1.03(1- \\
1.07)\end{array}$ & .03 & $\begin{array}{l}1.01(0.98- \\
1.04)\end{array}$ & .53 & $\begin{array}{l}1.04(1.03- \\
1.05)\end{array}$ & $<.001$ & $\begin{array}{l}0.96 \\
(0.95- \\
0.98)\end{array}$ & $<.001$ \\
\hline $\begin{array}{l}\text { Multivariate } \\
\text { model }\end{array}$ & $\begin{array}{l}0.22 \\
(0.19- \\
0.26)\end{array}$ & $<.001$ & $\begin{array}{l}1.04 \\
(1.02- \\
1.07)\end{array}$ & .002 & $\begin{array}{l}1.01(0.99- \\
1.04)\end{array}$ & .30 & $\begin{array}{l}0.99(0.97- \\
1.02)\end{array}$ & .69 & $\begin{array}{l}1.04(1.03- \\
1.05)\end{array}$ & $<.001$ & $\begin{array}{l}0.96 \\
(0.95- \\
0.98)\end{array}$ & $<.001$ \\
\hline
\end{tabular}

${ }^{a}$ Adherence to infection prevention and control procedures; adjusted for age, gender, ability to pay for medication, social status, type of health care personnel, type of health care facility, epidemic containment experience, and comorbidity in the multivariate model.

${ }^{\mathrm{b}}$ Adjusted for age, gender, marital status, ability to pay for medication, and social status in the multivariate model. The reference group is "less healthy diet" and the test group is "unchanged or healthier diet."

${ }^{\mathrm{c}}$ Adjusted for age, gender, marital status, social status, and type of health care personnel in the multivariate model. The reference group is "never, stopped, or less smoking," and the test group is "unchanged or more smoking."

${ }^{d}$ Adjusted for age, gender, social status, type of health care personnel, and epidemic containment experience in the multivariate model. The reference group is "never, stopped, or less drinking," and the test group is "unchanged or more drinking."

eAdjusted for age, gender, ability to pay for medication, social status, type of health care personnel, type of health care facility, and epidemic containment experience in the multivariate model. The reference group is "never, stopped, or less physical activity," and the test group is "unchanged or more physical activity."

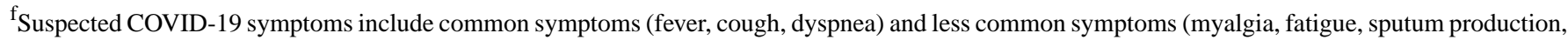
confusion, headache, sore throat, rhinorrhea, chest pain, hemoptysis, diarrhea, and nausea/vomiting). Adjusted for age, gender, marital status, ability to pay for medications, social status, type of health care personnel, and comorbidity in the multivariate model.

${ }^{\mathrm{B}} \mathrm{B}$ : unstandardized regression coefficient.

${ }^{\mathrm{h}} \mathrm{OR}$ : odds ratio.

\section{Association Between HL and eHealth Literacy and Lifestyle Changes}

The results of the multivariate logistic regression analysis shown in Table 5 indicated that HCWs with higher HL (OR 1.04, 95\% CI 1.01-1.06, $P=.001$ ) and higher eHEALS scores (OR 1.04, $95 \%$ CI 1.02-1.07, $P=.002$ ) had a higher likelihood of eating at an "unchanged or healthier" level after being adjusted for age, gender, marital status, ability to pay for medication, and social status. These adjusted factors had associations with eating behavior changes at $P<.20$ (Table 2 in Multimedia Appendix
1). In addition, HCWs with higher HL scores (OR 1.03, 95\% CI 1.02-1.03, $P<.001)$ and higher eHEALS scores (OR 1.04, 95\% CI 1.03-1.05, $P<.001$ ) had a higher likelihood of doing physical activity at an "unchanged or more" level after adjusting for age, gender, ability to pay for medication, social status, profession, type of health care facility, and epidemic containment experience. These adjusted factors had associations with physical activity at $P<.20$ (Table 2 in Multimedia Appendix 1). No association was found between HL and eHEALS scores and smoking or drinking behaviors. 


\section{Associations of HL and eHealth Literacy With Suspected COVID-19 Symptoms}

As shown in Table 5, HCWs with higher HL scores (OR 0.97, 95\% CI 0.96-0.98, $P<.001)$ and higher eHEALS scores (OR $0.96,95 \%$ CI $0.95-0.98, P<.001)$ had a lower likelihood of having suspected COVID-19 symptoms after adjusting for age, gender, marital status, ability to pay for medication, social status, profession, and comorbidities. These adjusted factors had associations with suspected COVID-19 symptoms at $P<.20$ (Table 2 in Multimedia Appendix 1).

\section{Discussion}

\section{Principal Findings}

The eHEALS questionnaire was found to be valid and reliable for assessing the eHealth literacy of HCWs. The tool was found to have satisfactory construct validity, convergent validity, criterion validity, and reliability. There was no flooring effect. A marginal ceiling effect was found. A high percentage of participants with the highest possible score was also observed in a previous study that validated the digital health literacy scale [64]. This could be because HCWs have been recognized as a group that has a higher standard of health knowledge and skills than the average person. Therefore, it is reasonable that many participants had a higher or maximum score.

In this study, male HCWs had higher HL and eHEALS scores. This finding is inconsistent with a previous study conducted among the general population in Europe [65], in which men had lower HL scores than women. Another study conducted among the general population in Taiwan showed that men also had lower scores of HL and digital health diet literacy than women [66]. In addition, eHealth literacy was found to be higher in women than men in the general population in Hungary [67] and in health care professionals in Ethiopia [68]. This inconsistency could be explained by the fact that although the total HCW population had fewer males $(32.9 \%$ versus $67.1 \%$ female), the proportion of male doctors (797/1453 or $54.9 \%)$ was higher than that of female doctors $(656 / 1453$ or $45.1 \%)$.

Doctors had higher HL and eHEALS scores than other HCWs in this study. Similarly, HCWs with epidemic containment experience had higher HL and eHEALS scores in this study. Among HCWs, doctors receive longer professional training and they have been recognized as the group with the highest level of ability to find, understand, justify, and use health-related information. In addition, they are involved in educating and counseling other HCWs and patients [69]. HCWs who were involved in containing previous outbreaks or epidemics logically had better health knowledge and skills that made their HL and eHEALS scores higher. HL was found to be a strategic approach for improving communication between patients and HCWs [41], further contributing to the containment of the pandemic and its consequences [45].

HCWs with a better ability to pay for medication had higher HL and eHEALS scores. A positive association between the ability to pay for medication was prominently found in many previous studies [51,52,66,70,71]. In addition, HCWs with middle or high social status had higher HL scores. This was similar to the findings of previous studies conducted in Asia [51,52,66,70-72] and Europe [65]. This implies that socioeconomic factors are independent indictors that should be taken into consideration in intervention development to improve HL and eHEALS scores.

In this study, HCWs with higher HL or eHEALS scores had better adherence to IPC procedures. This is the first study investigating these associations. In the literature, higher HL scores were found to be associated with better adherence to therapies among people with chronic diseases [73-76]. Receiving COVID-19-related information online during the pandemic could be beneficial for various preventive behaviors [77]. Therefore, more efforts are needed to increase the distribution of accurate information and encourage appropriate behaviors [78]. HCWs should be aware of and strictly adhere to the guidelines for IPC to contain the COVID-19 pandemic.

Our study found that HCWs with higher HL and eHEALS scores had a lower likelihood of suspected COVID-19 symptoms. It has been noted that individuals with higher HL scores had better health status [71,79] and well-being [80,81]. This could be further explained by the fact that higher HL and eHEALS scores were associated with healthier eating and physical activity behaviors in this study. In a previous study, we also found that medical and nursing students with higher digital healthy diet literacy had healthier eating behaviors during the pandemic [82]. In addition, eHealth literacy was found to be associated with key issues related to seeking out exercise regime information and maintaining a healthy diet [83]. In addition, it has been reported that people with higher HL scores had healthier behaviors (eg, exercise, balanced diet) [66,84], further protecting and improving their health and well-being [85].

This study has some limitations. First, the study was conducted online and the suspected COVID-19 symptoms were self-reported; therefore, we cannot confirm COVID-19 cases and exclude them from our study. Fortunately, there were no new confirmed cases during the data collection period [86]. Second, during the lockdown period, people were ordered to stay at home and only go out to buy food or medicine, for emergencies, or to work at businesses producing and providing essential goods and services. This may cause a measurement bias in the application domain of HL, as the questionnaire cannot distinguish between an individual's ability and restrictions during lockdown (eg, on questions such as "on a scale from very easy to very difficult, how easy would you say it is to join a sports club or exercise class if you want to?"). Third, a cross-sectional design was used, which cannot provide evidence of the causal relationships among studied variables. Furthermore, the convenient sampling technique used may limit the generalizability of the findings. In addition, the differences between some categories were not examined due to small sample sizes (eg, widowed/separated/divorced participants [140/5209, $2.7 \%$ ] or those with high social status 63/5209, 1.2\%]). However, we surveyed a relatively large sample of HCWs from 15 hospitals and health centers across Vietnam, which can help with exploring associations in future research and provides evidence for potential interventions. 


\section{Conclusions}

The eHEALS questionnaire is a valid and reliable tool for assessing eHealth literacy among HCWs. HL and eHealth literacy were significantly higher in men, those with better ability to pay for medication, doctors, and those with previous epidemic containment experience. Both HL and eHealth literacy were associated with better adherence to IPC procedures, healthier lifestyles (eg, healthier eating behavior and more physical activity during the pandemic), and a lower likelihood of having suspected COVID-19 symptoms. Integrative and multidisciplinary approaches are required to improve HCWs' HL and eHealth literacy, which could help improve adherence to IPC measures, promote healthy behaviors, and protect the health of HCWs. This would further contribute to containing the COVID-19 pandemic and minimizing its consequences.

\section{Acknowledgments}

We appreciate and acknowledge the participation of HCWs from the selected hospitals and health centers. This work was supported by Military Hospital 103, Vietnam, and Taipei Medical University, Taiwan (108-3805-022-400).

\section{Authors' Contributions}

BND, TVT, and TVD analyzed the data and drafted the manuscript. BND, TVT, DTP, HCN, TTPN, HCN, THH, HKD, MVT, TVD, HQN, TTN, NPTN, CQT, KVT, TTD, HXP, LVN, KTN, PWC, and TVD contributed to conceptualization, investigation, methodology, validation, and manuscript revision. BND, TVT, DTP, HCN, TTPN, HCN, THH, HKD, MVT, TVD, HQN, TTN, NPTN, CQT, KVT, TTD, HXP, LVN, KTN, and TVD conducted data curation. All authors gave final approval of the manuscript.

\section{Conflicts of Interest}

None declared.

\section{Multimedia Appendix 1}

Supplementary data.

[DOCX File , 38 KB-Multimedia Appendix 1]

\section{References}

1. Greenberg N, Docherty M, Gnanapragasam S, Wessely S. Managing mental health challenges faced by healthcare workers during covid-19 pandemic. BMJ 2020 Mar 26;368:m1211. [doi: 10.1136/bmj.m1211] [Medline: 32217624]

2. Bassetti M, Vena A, Giacobbe DR. The novel Chinese coronavirus (2019-nCoV) infections: Challenges for fighting the storm. Eur J Clin Invest 2020 Mar;50(3):e13209. [doi: 10.1111/eci.13209] [Medline: 32003000]

3. Phelan AL, Katz R, Gostin LO. The Novel Coronavirus Originating in Wuhan, China: Challenges for Global Health Governance. JAMA 2020 Feb 25;323(8):709-710 [FREE Full text] [doi: 10.1001/jama.2020.1097] [Medline: 31999307]

4. Rubin R. The Challenge of Preventing COVID-19 Spread in Correctional Facilities. JAMA 2020 May 12;323(18):1760-1761. [doi: 10.1001/jama.2020.5427] [Medline: 32259189]

5. Xiang Y, Jin Y, Cheung T. Joint International Collaboration to Combat Mental Health Challenges During the Coronavirus Disease 2019 Pandemic. JAMA Psychiatry 2020 Oct 01;77(10):989-990. [doi: 10.1001/jamapsychiatry.2020.1057] [Medline: 32275289]

6. Campion J, Javed A, Sartorius N, Marmot M. Addressing the public mental health challenge of COVID-19. The Lancet Psychiatry 2020 Aug;7(8):657-659. [doi: 10.1016/s2215-0366(20)30240-6]

7. Nicola M, Alsafi Z, Sohrabi C, Kerwan A, Al-Jabir A, Iosifidis C, et al. The socio-economic implications of the coronavirus pandemic (COVID-19): A review. Int J Surg 2020 Jun;78:185-193 [FREE Full text] [doi: 10.1016/j.ijsu.2020.04.018] [Medline: $\underline{32305533}$ ]

8. Clark A, Jit M, Warren-Gash C, Guthrie B, Wang HHX, Mercer SW, Centre for the Mathematical Modelling of Infectious Diseases COVID-19 working group. Global, regional, and national estimates of the population at increased risk of severe COVID-19 due to underlying health conditions in 2020: a modelling study. Lancet Glob Health 2020 Aug;8(8):e1003-e1017 [FREE Full text] [doi: 10.1016/S2214-109X(20)30264-3] [Medline: 32553130]

9. Banerjee A, Pasea L, Harris S, Gonzalez-Izquierdo A, Torralbo A, Shallcross L, et al. Estimating excess 1-year mortality associated with the COVID-19 pandemic according to underlying conditions and age: a population-based cohort study. Lancet 2020 May 30;395(10238):1715-1725 [FREE Full text] [doi: 10.1016/S0140-6736(20)30854-0] [Medline: 32405103]

10. Moradian N, Ochs HD, Sedikies C, Hamblin MR, Camargo CA, Martinez JA, et al. The urgent need for integrated science to fight COVID-19 pandemic and beyond. J Transl Med 2020 May 19;18(1):205 [FREE Full text] [doi: 10.1186/s12967-020-02364-2] [Medline: 32430070]

11. Holmes EA, O'Connor RC, Perry VH, Tracey I, Wessely S, Arseneault L, et al. Multidisciplinary research priorities for the COVID-19 pandemic: a call for action for mental health science. Lancet Psychiatry 2020 Jun;7(6):547-560 [FREE Full text] [doi: 10.1016/S2215-0366(20)30168-1] [Medline: 32304649] 
12. Nazir M, Hussain I, Tian J, Akram S, Mangenda Tshiaba S, Mushtaq S, et al. A Multidimensional Model of Public Health Approaches Against COVID-19. Int J Environ Res Public Health 2020 May 26;17(11):3780 [FREE Full text] [doi: 10.3390/ijerph17113780] [Medline: $\underline{\text { 32466581] }}$

13. Betsch C. How behavioural science data helps mitigate the COVID-19 crisis. Nat Hum Behav 2020 May 27;4(5):438-438 [FREE Full text] [doi: 10.1038/s41562-020-0866-1] [Medline: 32221514]

14. Bavel JJV, Baicker K, Boggio PS, Capraro V, Cichocka A, Cikara M, et al. Using social and behavioural science to support COVID-19 pandemic response. Nat Hum Behav 2020 May 30;4(5):460-471. [doi: 10.1038/s41562-020-0884-z] [Medline: 32355299]

15. Paakkari L, Okan O. COVID-19: health literacy is an underestimated problem. Lancet Public Health 2020 May;5(5):e249-e250 [FREE Full text] [doi: 10.1016/S2468-2667(20)30086-4] [Medline: 32302535]

16. Chong YY, Cheng HY, Chan HYL, Chien WT, Wong SYS. COVID-19 pandemic, infodemic and the role of eHealth literacy. Int J Nurs Stud 2020 Aug;108:103644 [FREE Full text] [doi: 10.1016/j.ijnurstu.2020.103644] [Medline: 32447127]

17. PM orders strict nationwide social distancing rules, starting April 1. Vietnam Law Magazine. 2020. URL: https:/ /vietnamlawmagazine.vn/pm-orders-strict-nationwide-social-distancing-rules-starting-april-1-27108.html [accessed 2020-03-31]

18. Hao F, Tan W, Jiang L, Zhang L, Zhao X, Zou Y, et al. Do psychiatric patients experience more psychiatric symptoms during COVID-19 pandemic and lockdown? A case-control study with service and research implications for immunopsychiatry. Brain Behav Immun 2020 Jul;87:100-106 [FREE Full text] [doi: 10.1016/j.bbi.2020.04.069] [Medline: 32353518]

19. Ozamiz-Etxebarria N, Idoiaga Mondragon N, Dosil Santamaría M, Picaza Gorrotxategi M. Psychological Symptoms During the Two Stages of Lockdown in Response to the COVID-19 Outbreak: An Investigation in a Sample of Citizens in Northern Spain. Front Psychol 2020;11:1491 [FREE Full text] [doi: 10.3389/fpsyg.2020.01491] [Medline: 32625157]

20. Di Renzo L, Gualtieri P, Pivari F, Soldati L, Attinà A, Cinelli G, et al. Eating habits and lifestyle changes during COVID-19 lockdown: an Italian survey. J Transl Med 2020 Jun 08;18(1):229 [FREE Full text] [doi: 10.1186/s12967-020-02399-5] [Medline: 32513197]

21. Ammar A, Brach M, Trabelsi K, Chtourou H, Boukhris O, Masmoudi L, et al. Effects of COVID-19 Home Confinement on Eating Behaviour and Physical Activity: Results of the ECLB-COVID19 International Online Survey. Nutrients 2020 May 28;12(6):1583 [FREE Full text] [doi: 10.3390/nu12061583] [Medline: 32481594]

22. Sidor A, Rzymski P. Dietary Choices and Habits during COVID-19 Lockdown: Experience from Poland. Nutrients 2020 Jun 03;12(6):1657 [FREE Full text] [doi: 10.3390/nu12061657] [Medline: 32503173]

23. Rodríguez-Pérez C, Molina-Montes E, Verardo V, Artacho R, García-Villanova B, Guerra-Hernández EJ, et al. Changes in Dietary Behaviours during the COVID-19 Outbreak Confinement in the Spanish COVIDiet Study. Nutrients 2020 Jun 10;12(6):1730 [FREE Full text] [doi: 10.3390/nu12061730] [Medline: 32531892]

24. Wardell J, Kempe T, Rapinda K, Single A, Bilevicius E, Frohlich J, et al. Drinking to Cope During COVID-19 Pandemic: The Role of External and Internal Factors in Coping Motive Pathways to Alcohol Use, Solitary Drinking, and Alcohol Problems. Alcohol Clin Exp Res 2020 Sep 01:1. [doi: 10.1111/acer.14425] [Medline: 32870516]

25. Gong K, Xu Z, Cai Z, Chen Y, Wang Z. Internet Hospitals Help Prevent and Control the Epidemic of COVID-19 in China: Multicenter User Profiling Study. J Med Internet Res 2020 Apr 14;22(4):e18908 [FREE Full text] [doi: 10.2196/18908] [Medline: 32250962]

26. Sesagiri Raamkumar A, Tan S, Wee H. Measuring the Outreach Efforts of Public Health Authorities and the Public Response on Facebook During the COVID-19 Pandemic in Early 2020: Cross-Country Comparison. J Med Internet Res 2020 May 19;22(5):e19334 [FREE Full text] [doi: 10.2196/19334] [Medline: 32401219]

27. Shen C, Chen A, Luo C, Zhang J, Feng B, Liao W. Using Reports of Symptoms and Diagnoses on Social Media to Predict COVID-19 Case Counts in Mainland China: Observational Infoveillance Study. J Med Internet Res 2020 May 28;22(5):e19421 [FREE Full text] [doi: 10.2196/19421] [Medline: 32452804]

28. Abd-Alrazaq A, Alhuwail D, Househ M, Hamdi M, Shah Z. Top Concerns of Tweeters During the COVID-19 Pandemic: Infoveillance Study. J Med Internet Res 2020 Apr 21;22(4):e19016 [FREE Full text] [doi: 10.2196/19016] [Medline: 32287039]

29. Liu Q, Zheng Z, Zheng J, Chen Q, Liu G, Chen S, et al. Health Communication Through News Media During the Early Stage of the COVID-19 Outbreak in China: Digital Topic Modeling Approach. J Med Internet Res 2020 Apr 28;22(4):e19118 [FREE Full text] [doi: 10.2196/19118] [Medline: 32302966]

30. Liao Q, Yuan J, Dong M, Yang L, Fielding R, Lam WWT. Public Engagement and Government Responsiveness in the Communications About COVID-19 During the Early Epidemic Stage in China: Infodemiology Study on Social Media Data. J Med Internet Res 2020 May 26;22(5):e18796 [FREE Full text] [doi: 10.2196/18796] [Medline: 32412414]

31. Ahmad AR, Murad HR. The Impact of Social Media on Panic During the COVID-19 Pandemic in Iraqi Kurdistan: Online Questionnaire Study. J Med Internet Res 2020 May 19;22(5):e19556 [FREE Full text] [doi: 10.2196/19556] [Medline: $\underline{32369026]}$

32. Bao Y, Sun Y, Meng S, Shi J, Lu L. 2019-nCoV epidemic: address mental health care to empower society. Lancet 2020 Feb 22;395(10224):e37-e38 [FREE Full text] [doi: 10.1016/S0140-6736(20)30309-3] [Medline: 32043982] 
33. Shimizu K. 2019-nCoV, fake news, and racism. Lancet 2020 Feb 29;395(10225):685-686 [FREE Full text] [doi: 10.1016/S0140-6736(20)30357-3] [Medline: 32059801]

34. Brooks SK, Webster RK, Smith LE, Woodland L, Wessely S, Greenberg N, et al. The psychological impact of quarantine and how to reduce it: rapid review of the evidence. Lancet 2020 Mar 14;395(10227):912-920 [FREE Full text] [doi: 10.1016/S0140-6736(20)30460-8] [Medline: 32112714]

35. Diviani N, van den Putte B, Giani S, van Weert JC. Low health literacy and evaluation of online health information: a systematic review of the literature. J Med Internet Res 2015 May 07;17(5):e112 [FREE Full text] [doi: 10.2196/jmir.4018] [Medline: 25953147]

36. Norman CD, Skinner HA. eHealth Literacy: Essential Skills for Consumer Health in a Networked World. J Med Internet Res 2006 Jun 16;8(2):e9 [FREE Full text] [doi: 10.2196/jmir.8.2.e9] [Medline: 16867972]

37. Park HW, Park S, Chong M. Conversations and Medical News Frames on Twitter: Infodemiological Study on COVID-19 in South Korea. J Med Internet Res 2020 May 05;22(5):e18897. [doi: 10.2196/18897] [Medline: $\underline{32325426]}$

38. Ko N, Lu W, Chen Y, Li D, Wang P, Hsu S, et al. COVID-19-related information sources and psychological well-being: An online survey study in Taiwan. Brain Behav Immun 2020 Jul;87:153-154 [FREE Full text] [doi: 10.1016/j.bbi.2020.05.019] [Medline: 32389702]

39. Mackert M, Ball J, Lopez N. Health literacy awareness training for healthcare workers: improving knowledge and intentions to use clear communication techniques. Patient Educ Couns 2011 Dec;85(3):e225-e228. [doi: 10.1016/j.pec.2011.02.022] [Medline: 21474264]

40. Yunyongying P, Rich M, Jokela J. Patient-Centered Performance Metrics. JAMA 2019 May 14;321(18):1829. [doi: 10.1001/jama.2019.2056] [Medline: $\underline{31087017]}$

41. McKinn S, Linh DT, Foster K, McCaffery K. Communication Between Health Workers and Ethnic Minorities in Vietnam. Health Lit Res Pract 2017 Oct 01;1(4):e163-e172 [FREE Full text] [doi: 10.3928/24748307-20170629-01] [Medline: 31294262]

42. Tavakoly Sany SB, Behzhad F, Ferns G, Peyman N. Communication skills training for physicians improves health literacy and medical outcomes among patients with hypertension: a randomized controlled trial. BMC Health Serv Res 2020 Jan 23;20(1):60 [FREE Full text] [doi: 10.1186/s12913-020-4901-8] [Medline: $\underline{\text { 31973765] }}$

43. Wang M, Hung L, Lo Y. Glycemic control in type 2 diabetes: role of health literacy and shared decision-making. Patient Prefer Adherence 2019;13:871-879 [FREE Full text] [doi: 10.2147/PPA.S202110] [Medline: 31213781]

44. Redelmeier DA, Shafir E. Pitfalls of judgment during the COVID-19 pandemic. Lancet Public Health 2020 Jun;5(6):e306-e308. [doi: 10.1016/S2468-2667(20)30096-7] [Medline: 32334647]

45. Sentell T, Vamos S, Okan O. Interdisciplinary Perspectives on Health Literacy Research Around the World: More Important Than Ever in a Time of COVID-19. Int J Environ Res Public Health 2020 Apr 26;17(9):3010 [FREE Full text] [doi: 10.3390/ijerph17093010] [Medline: 32357457]

46. Govt extends social distancing for at least one week in 28 localities. VGP News. 2020. URL: http://news.chinhphu.vn/ Home/Govt-extends-social-distancing-for-at-least-one-week-in-28-localities/20204/39735.vgp [accessed 2020-04-15]

47. Govt to gradually ease COVID-19 control measure in cautious manner. VGP News. 2020. URL: http://primeminister. chinhphu.vn/Home/Govt-to-gradually-ease-COVID19-control-measure-in-cautious-manner/20204/3759.vgp [accessed 2020-04-20]

48. Quan H, Li B, Couris CM, Fushimi K, Graham P, Hider P, et al. Updating and validating the Charlson comorbidity index and score for risk adjustment in hospital discharge abstracts using data from 6 countries. Am J Epidemiol 2011 Mar 15;173(6):676-682. [doi: 10.1093/aje/kwq433] [Medline: 21330339]

49. Charlson ME, Pompei P, Ales KL, MacKenzie C. A new method of classifying prognostic comorbidity in longitudinal studies: development and validation. J Chronic Dis 1987;40(5):373-383. [doi: 10.1016/0021-9681(87)90171-8] [Medline: $\underline{3558716}$ ]

50. Duong TV, Aringazina A, Kayupova G, Nurjanah, Pham TV, Pham KM, et al. Development and Validation of a New Short-Form Health Literacy Instrument (HLS-SF12) for the General Public in Six Asian Countries. Health Lit Res Pract 2019 Apr;3(2):e91-e102 [FREE Full text] [doi: 10.3928/24748307-20190225-01] [Medline: $\underline{31294310}$ ]

51. Duong TV, Nguyen TTP, Pham KM, Nguyen KT, Giap MH, Tran TDX, et al. Validation of the Short-Form Health Literacy Questionnaire (HLS-SF12) and Its Determinants among People Living in Rural Areas in Vietnam. Int J Environ Res Public Health 2019 Sep 11;16(18):3346 [FREE Full text] [doi: 10.3390/ijerph16183346] [Medline: $\underline{31514271]}$

52. Van Hoa H, Giang HT, Vu PT, Van Tuyen D, Khue PM. Factors Associated with Health Literacy among the Elderly People in Vietnam. Biomed Res Int 2020 Mar 27;2020:3490635-3490637. [doi: 10.1155/2020/3490635] [Medline: 32309429]

53. Nguyen HC, Nguyen MH, Do BN, Tran CQ, Nguyen TTP, Pham KM, et al. People with Suspected COVID-19 Symptoms Were More Likely Depressed and Had Lower Health-Related Quality of Life: The Potential Benefit of Health Literacy. J Clin Med 2020 Mar 31;9(4):965 [FREE Full text] [doi: 10.3390/jcm9040965] [Medline: 32244415]

54. Nguyen HT, Do BN, Pham KM, Kim GB, Dam HT, Nguyen TT, et al. Fear of COVID-19 Scale-Associations of Its Scores with Health Literacy and Health-Related Behaviors among Medical Students. Int J Environ Res Public Health 2020 Jun 11;17(11):4164. [doi: 10.3390/ijerph17114164] [Medline: 32545240] 
55. HLS-EU Consortium. Comparative report of health literacy in eight EU member states. The European Health Literacy Project. 2009. URL: https://www.healthliteracyeurope.net/hls-eu [accessed 2012-10-22]

56. Norman CD, Skinner HA. eHEALS: The eHealth Literacy Scale. J Med Internet Res 2006 Nov 14;8(4):e27 [FREE Full text] [doi: 10.2196/jmir.8.4.e27] [Medline: 17213046]

57. World Health Organization. Risk assessment and management of exposure of health care workers in the context of COVID-19: Interim guidance. 2020. URL: https://www.who.int/publications/i/item/

risk-assessment-and-management-of-exposure-of-health-care-workers-in-the-context-of-covid-19-interim-guidance [accessed 2020-03-19]

58. Editorial Team. Overview of novel coronavirus (2019-nCoV). BMJ Best Practice. 2020. URL: https://bestpractice.bmj.com/ topics/en-gb/3000165 [accessed 2020-02-10]

59. Field A. Discovering statistics using IBM SPSS statistics. Washington DC, USA: Sage Publications Ltd; 2013.

60. Kimberlin C, Winterstein A. Validity and reliability of measurement instruments used in research. Am J Health Syst Pharm 2008 Dec 01;65(23):2276-2284. [doi: 10.2146/ajhp070364] [Medline: 19020196]

61. Terwee CB, Bot SDM, de Boer MR, van der Windt DAWM, Knol DL, Dekker J, et al. Quality criteria were proposed for measurement properties of health status questionnaires. J Clin Epidemiol 2007 Jan;60(1):34-42. [doi: 10.1016/j.jclinepi.2006.03.012] [Medline: 17161752]

62. Cronbach LJ, Shavelson RJ. My Current Thoughts on Coefficient Alpha and Successor Procedures. Educational and Psychological Measurement 2016 Jul 02;64(3):391-418. [doi: 10.1177/0013164404266386]

63. Maldonado G, Greenland S. Simulation study of confounder-selection strategies. Am J Epidemiol 1993 Dec 01;138(11):923-936. [doi: 10.1093/oxfordjournals.aje.a116813] [Medline: $\underline{\text { 8256780] }}$

64. van der Vaart R, Drossaert C. Development of the Digital Health Literacy Instrument: Measuring a Broad Spectrum of Health 1.0 and Health 2.0 Skills. J Med Internet Res 2017 Jan 24;19(1):e27 [FREE Full text] [doi: 10.2196/jmir.6709] [Medline: 28119275]

65. Sørensen K, Pelikan JM, Röthlin F, Ganahl K, Slonska Z, Doyle G, HLS-EU Consortium. Health literacy in Europe: comparative results of the European health literacy survey (HLS-EU). Eur J Public Health 2015 Dec 05;25(6):1053-1058 [FREE Full text] [doi: 10.1093/eurpub/ckv043] [Medline: 25843827]

66. Van Duong T, Chiu C, Lin C, Chen YC, Wong TC, Chang P, et al. E-healthy diet literacy scale and its relationship with behaviors and health outcomes in Taiwan. Health Promot Int 2020 Apr 08:E. [doi: 10.1093/heapro/daaa033] [Medline: 32267935]

67. Zrubka Z, Hajdu O, Rencz F, Baji P, Gulácsi L, Péntek M. Psychometric properties of the Hungarian version of the eHealth Literacy Scale. Eur J Health Econ 2019 Jun 16;20(Suppl 1):57-69 [FREE Full text] [doi: 10.1007/s10198-019-01062-1] [Medline: $\underline{\text { 31098883] }}$

68. Shiferaw KB, Mehari EA. Internet use and eHealth literacy among health-care professionals in a resource limited setting: a cross-sectional survey. Adv Med Educ Pract 2019;10:563-570 [FREE Full text] [doi: 10.2147/AMEP.S205414] [Medline: 31440113]

69. Palesy D, Jakimowicz S. Health literacy training for Australian home care workers: Enablers and barriers. Home Health Care Serv Q 2019 Apr 20;38(2):80-95. [doi: 10.1080/01621424.2019.1604458] [Medline: 31007141]

70. Duong T, Sørensen K, Pelikan JM, Van den Broucke S, Lin I, Lin Y, et al. Health-related behaviors moderate the association between age and self-reported health literacy among Taiwanese women. Women Health 2018 Jul 06;58(6):632-646. [doi: 10.1080/03630242.2017.1333074] [Medline: 28537772]

71. Kim D, Chang H. Key functional characteristics in designing and operating health information websites for user satisfaction: an application of the extended technology acceptance model. Int J Med Inform 2007;76(11-12):790-800. [doi: 10.1016/j.ijmedinf.2006.09.001] [Medline: 17049917]

72. Duong TV, Aringazina A, Baisunova G, Nurjanah, Pham TV, Pham KM, et al. Measuring health literacy in Asia: Validation of the HLS-EU-Q47 survey tool in six Asian countries. J Epidemiol 2017 Feb;27(2):80-86 [FREE Full text] [doi: 10.1016/j.je.2016.09.005] [Medline: 28142016]

73. Elisabeth Stømer U, Klopstad Wahl A, Gunnar Gøransson L, Hjorthaug Urstad K. Health Literacy in Kidney Disease: Associations with Quality of Life and Adherence. J Ren Care 2020 Jun 16;46(2):85-94. [doi: 10.1111/jorc.12314] [Medline: 31950601]

74. Huang Y, Shiyanbola OO, Chan H. A path model linking health literacy, medication self-efficacy, medication adherence, and glycemic control. Patient Educ Couns 2018 Nov;101(11):1906-1913. [doi: 10.1016/j.pec.2018.06.010] [Medline: 29935739]

75. Lor M, Koleck TA, Bakken S, Yoon S, Dunn Navarra A. Association Between Health Literacy and Medication Adherence Among Hispanics with Hypertension. J Racial Ethn Health Disparities 2019 Jun 3;6(3):517-524 [FREE Full text] [doi: 10.1007/s40615-018-00550-z] [Medline: 30607576]

76. Skoumalova I, Kolarcik P, Madarasova Geckova A, Rosenberger J, Majernikova M, Klein D, et al. Is Health Literacy of Dialyzed Patients Related to Their Adherence to Dietary and Fluid Intake Recommendations? Int J Environ Res Public Health 2019 Nov 05;16(21):4295 [FREE Full text] [doi: 10.3390/ijerph16214295] [Medline: 31694265] 
77. Li S, Feng B, Liao W, Pan W. Internet Use, Risk Awareness, and Demographic Characteristics Associated With Engagement in Preventive Behaviors and Testing: Cross-Sectional Survey on COVID-19 in the United States. J Med Internet Res 2020 Jun 16;22(6):e19782 [FREE Full text] [doi: 10.2196/19782] [Medline: 32501801]

78. Alsan M, Stantcheva S, Yang D, Cutler D. Disparities in Coronavirus 2019 Reported Incidence, Knowledge, and Behavior Among US Adults. JAMA Netw Open 2020 Jun 01;3(6):e2012403 [FREE Full text] [doi:

10.1001/jamanetworkopen.2020.12403] [Medline: $\underline{\text { 32556260] }}$

79. Duong VT, Lin I, Sorensen K, Pelikan JM, Van Den Broucke S, Lin Y, et al. Health Literacy in Taiwan: A Population-Based Study. Asia Pac J Public Health 2015 Nov 29;27(8):871-880. [doi: 10.1177/1010539515607962] [Medline: 26419635]

80. Watson R. Europeans with poor "health literacy" are heavy users of health services. BMJ 2011 Nov 29;343(nov29 4):d7741-d7741. [doi: 10.1136/bmj.d7741] [Medline: 22127773]

81. Greenhalgh T. Health literacy: towards system level solutions. BMJ 2015 Feb 24;350(feb24 13):h1026-h1026. [doi: 10.1136/bmj.h1026] [Medline: 25712067]

82. Duong TV, Pham KM, Do BN, Kim GB, Dam HTB, Le VT, et al. Digital Healthy Diet Literacy and Self-Perceived Eating Behavior Change during COVID-19 Pandemic among Undergraduate Nursing and Medical Students: A Rapid Online Survey. Int J Environ Res Public Health 2020 Sep 30;17(19):7185 [FREE Full text] [doi: 10.3390/ijerph17197185] [Medline: 33008102]

83. Britt RK, Collins WB, Wilson K, Linnemeier G, Englebert AM. eHealth Literacy and Health Behaviors Affecting Modern College Students: A Pilot Study of Issues Identified by the American College Health Association. J Med Internet Res 2017 Dec 19;19(12):e392 [FREE Full text] [doi: 10.2196/jmir.3100] [Medline: 29258979]

84. Mitsutake S, Shibata A, Ishii K, Oka K. Associations of eHealth Literacy With Health Behavior Among Adult Internet Users. J Med Internet Res 2016 Jul 18;18(7):e192 [FREE Full text] [doi: 10.2196/jmir.5413] [Medline: 27432783]

85. Owen L, Corfe B. The role of diet and nutrition on mental health and wellbeing. Proc Nutr Soc 2017 Nov;76(4):425-426. [doi: 10.1017/S0029665117001057] [Medline: 28707609]

86. Ministry of Health. Coronavirus disease (COVID-19) outbreak in Vietnam. 2020. URL: https://ncov.moh.gov.vn/ [accessed 2020-05-04]

\author{
Abbreviations \\ B: unstandardized regression coefficient \\ eHEALS: eHealth Literacy Scale \\ HCWs: health care workers \\ HL: health literacy \\ HLS-SF12: 12-item short-form health literacy questionnaire \\ IPC: infection prevention and control \\ KMO: Kaiser-Meyer Olkin Measure \\ PCA: principal component analysis \\ PPE: personal protective equipment \\ WHO: World Health Organization
}

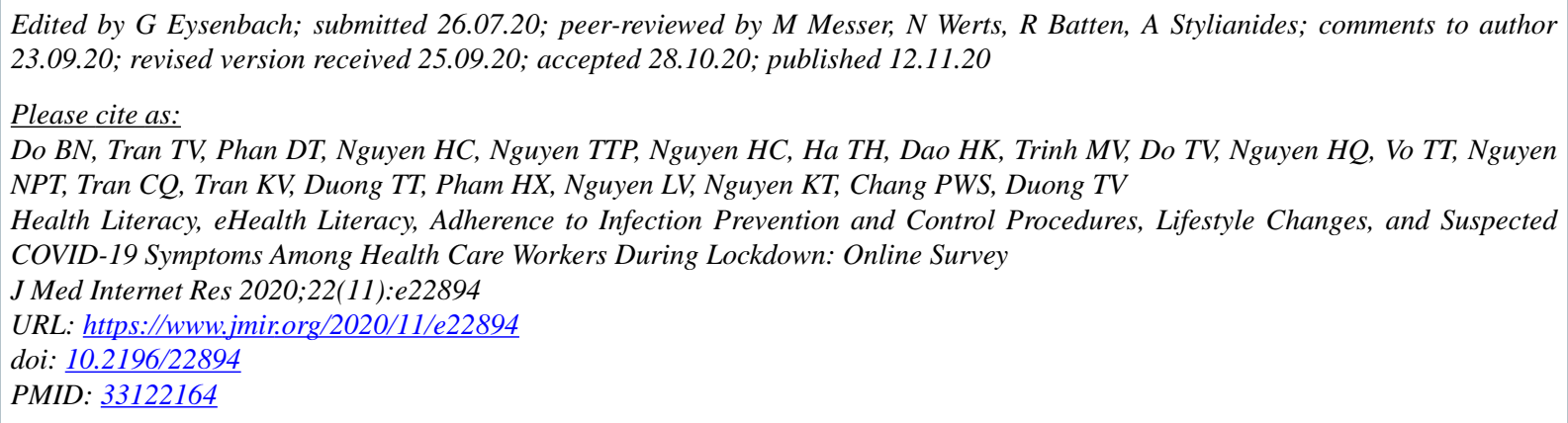

CBinh N Do, Tien V Tran, Dung T Phan, Hoang C Nguyen, Thao T P Nguyen, Huu C Nguyen, Tung H Ha, Hung K Dao, Manh V Trinh, Thinh V Do, Hung Q Nguyen, Tam T Vo, Nhan P T Nguyen, Cuong Q Tran, Khanh V Tran, Trang T Duong, Hai X Pham, Lam V Nguyen, Kien T Nguyen, Peter W S Chang, Tuyen Van Duong. Originally published in the Journal of Medical Internet Research (http://www.jmir.org), 12.11.2020. This is an open-access article distributed under the terms of the Creative Commons Attribution License (https://creativecommons.org/licenses/by/4.0/), which permits unrestricted use, distribution, and 
reproduction in any medium, provided the original work, first published in the Journal of Medical Internet Research, is properly cited. The complete bibliographic information, a link to the original publication on http://www.jmir.org/, as well as this copyright and license information must be included. 